\title{
ERRATUM
}

Staffan Uhlén · Jonas Lindblom · Anna Kindlundh

Paul Mugisha $\cdot$ Fred Nyberg

\section{Nandrolone treatment decreases the level of rat kidney $\alpha_{1 \mathrm{~B}}$-adrenoceptors}

Published online: 29 October 2003

(C) Springer-Verlag 2003

\section{Naunyn-Schmiedeberg's Arch Pharmacol (2003) 368:91-98}

In Table 1, two values were inadvertently transposed. The correct table is given here:

Table 1

\begin{tabular}{lllllllll}
\hline & $\begin{array}{l}\alpha_{1 \mathrm{~A}} \mathrm{pK}_{\mathrm{d}} \\
{\left[{ }^{3} \mathrm{H}\right]-\mathrm{prazosin}}\end{array}$ & $\begin{array}{l}\alpha_{1 \mathrm{~B}} \mathrm{pK}_{\mathrm{d}} \\
{\left[{ }^{3} \mathrm{H}\right]-\mathrm{prazosin}}\end{array}$ & $\begin{array}{l}\alpha_{1 \mathrm{~A}} \\
(\mathrm{fmol} / \mathrm{mg})\end{array}$ & $\begin{array}{l}\alpha_{1 \mathrm{~B}} \\
(\mathrm{fmol} / \mathrm{mg})\end{array}$ & $\begin{array}{l}\alpha_{1 \mathrm{~B}}(\% \text { in } \\
\text { individual } \\
\text { experiments })\end{array}$ & $\alpha_{1 \mathrm{~A}} \mathrm{pK}_{\mathrm{i}} 5-\mathrm{MU}$ & $\alpha_{1 \mathrm{~B}} \mathrm{pK} \mathrm{i}_{\mathrm{i}} 5-\mathrm{MU}$ & $(\mathrm{n})$ \\
\hline t-test & $p=0.46$ & $p=0.35$ & $p=0.19$ & $p<0.0001$ & $p<0.0001$ & $p=0.21$ & $p=0.24$ \\
control & $9.40 \pm 0.07$ & $\underline{9.63 \pm 0.09}$ & $44.4 \pm 3.2$ & $14.8 \pm 1.8$ & $25.0 \pm 2.8$ & $8.63 \pm 0.08$ & $6.66 \pm 0.17$ & 12 \\
$\begin{array}{l}\text { nandrolone } \\
\text { treated }\end{array}$ & $\underline{9.41 \pm 0.07}$ & $9.58 \pm 0.12$ & $48.6 \pm 3.6$ & $6.5 \pm 0.8$ & $12.4 \pm 1.6$ & $8.53 \pm 0.08$ & $6.41 \pm 0.30$ & 13 \\
\hline
\end{tabular}

The online version of the original article can be found at http://dx.doi.org/10.1007/s00210-003-0763-y

S. Uhlén · A. Kindlundh · P. Mugisha $\cdot$ F. Nyberg

Department of Pharmaceutical Biosciences, Uppsala University,

Box 591 BioMedical Centre, 75124 Uppsala, Sweden

J. Lindblom (®)

Department of Neuroscience, Division of Pharmacology,

Uppsala University, Box 593 BioMedical Centre,

75124 Uppsala, Sweden,

Fax: +46-18-511540,

e-mail: Jonas.Lindblom@neuro.uu.se 